\title{
VARIABLE EXPONENT WEIGHTED NORM INEQUALITY FOR GENERALIZED RIESZ POTENTIALS
}

\author{
Fumi-Yuki Maeda, Yoshihiro Mizuta and Tetsu Shimomura
}

4-24 Furue-higashi-machi, Nishi-ku, Hiroshima 733-0872, Japan; fymaeda@h6.dion.ne.jp

4-13-11 Hachi-Hon-Matsu-Minami, Higashi-Hiroshima 739-0144, Japan;

yomizuta@hiroshima-u.ac.jp

Hiroshima University, Graduate School of Education, Department of Mathematics

Higashi-Hiroshima 739-8524, Japan; tshimo@hiroshima-u.ac.jp

\begin{abstract}
Our aim in this paper is to establish variable exponent weighted norm inequalities for generalized Riesz potentials via norm inequalities in non-homogeneous central Herz-Morrey spaces.
\end{abstract}

\section{Introduction}

Let $I_{\alpha}(x)=|x|^{\alpha-N}$ be the Riesz kernel of order $\alpha(0<\alpha<N)$ on the Euclidean $N$-space $\mathbf{R}^{N}$. The classical Sobolev's inequality for Riesz potentials is

$$
\left\|I_{\alpha} f\right\|_{L^{p^{*}}\left(\mathbf{R}^{N}\right)} \leq C\|f\|_{L^{p}\left(\mathbf{R}^{N}\right)}
$$

for $f \in L^{p}\left(\mathbf{R}^{N}\right), 1<p<N / \alpha$, where $1 / p^{*}=1 / p-\alpha / N$. For weighted Lebesgue spaces $L^{p, w}$, Muckenhoupt-Wheeden [13] showed

$$
\left\|I_{\alpha} f\right\|_{L^{p^{*}, w^{p^{*} / p}\left(\mathbf{R}^{N}\right)}} \leq C\|f\|_{L^{p, w}\left(\mathbf{R}^{N}\right)}
$$

under certain conditions on the weight $w$. In case $w(x)=(1+|x|)^{-a}$, the condition is $N-N p<a<N-\alpha p$. These results have been extended to the case of variable exponent; see [1] and [4] for unweighted spaces, and [11], [12] and [14] for weighted spaces.

The above inequalities may be called of $L^{p}-L^{p^{*}}$ type. In [7], Kurokawa gave a $L^{p}-L^{p}$ type inequality

$$
\left\|I_{\alpha} f\right\|_{L^{p, a+p \alpha}\left(\mathbf{R}^{N}\right)} \leq C\|f\|_{L^{p, a}\left(\mathbf{R}^{N}\right)},
$$

where $L^{p, a}\left(\mathbf{R}^{N}\right)=L^{p, w}\left(\mathbf{R}^{N}\right)$ with $w(x)=(1+|x|)^{-a}(0<a<N-\alpha p)$. Kurokawa also gave similar inequalities for generalized Riesz potentials $I_{\alpha, k} f$, which are defined as

$$
I_{\alpha, k} f(x)=\int_{\mathbf{R}^{N}} I_{\alpha, k}(x, y) f(y) d y
$$

whenever the integral is well-defined, where

$$
I_{\alpha, k}(x, y)= \begin{cases}I_{\alpha}(x-y) & \text { for }|y|<1 \\ I_{\alpha}(x-y)-\sum_{|\mu| \leq k-1} \frac{x^{\mu}}{\mu !}\left(D^{\mu} I_{\alpha}\right)(-y) & \text { for }|y| \geq 1\end{cases}
$$

for integers $k \geq 1$.

https://doi.org/10.5186/aasfm.2018.4336

2010 Mathematics Subject Classification: Primary 46E30, 31B15.

Key words: Weighted norm inequality, variable exponent, Sobolev's inequality, Riesz potentials. 
Our aim of this paper is to extend Kurokawa's results to variable exponent case. To this end, we consider variable exponent non-homogeneous central Herz-Morrey spaces $\mathcal{H}^{p(\cdot), q, \omega}\left(\mathbf{R}^{N}\right)$ (whose definition will be given in Section 2) and we shall establish norm inequalities for the operators $f \rightarrow I_{\alpha} f$ and $f \rightarrow I_{\alpha, k} f$ from $\mathcal{H}^{p(\cdot), q, \omega}\left(\mathbf{R}^{N}\right)$ to $\mathcal{H}^{p(\cdot), q, \omega_{-\alpha}}\left(\mathbf{R}^{N}\right)\left(\omega_{-\alpha}(r)=r^{-\alpha} \omega(r)\right.$ for $\left.r \geq 1\right)$. Then the required results follow from the observation that $L^{p(\cdot), \omega}\left(\mathbf{R}^{N}\right)=\mathcal{H}^{p(\cdot), p(\infty), \omega^{1 / p(\infty)}}\left(\mathbf{R}^{N}\right)$.

Throughout this paper, let $C$ denote various positive constants independent of the variables in question.

\section{Preliminaries}

Throughout, let $p(\cdot)$ be a measurable function on $\mathbf{R}^{N}$ such that

$$
1 \leq p^{-}:=\operatorname{ess} \inf _{x} p(x) \leq \operatorname{ess} \sup _{x} p(x)=: p^{+}<\infty
$$

and assume that it is log-Hölder continuous at $\infty$ :

$$
|p(x)-p(\infty)| \leq \frac{c_{\infty}}{\log (e+|x|)} \quad \text { for all } x \in \mathbf{R}^{N}
$$

For a measurable set $\Omega \subset \mathbf{R}^{N}$, we consider the variable exponent Lebesgue space

$$
L^{p(\cdot)}(\Omega)=\left\{f \in L_{\mathrm{loc}}^{1}(\Omega):\|f\|_{L^{p(\cdot)}(\Omega)}<\infty\right\},
$$

where

$$
\|f\|_{L^{p(\cdot)}(\Omega)}=\inf \left\{\lambda>0: \int_{\Omega}\left(\frac{|f(x)|}{\lambda}\right)^{p(x)} d x \leq 1\right\} .
$$

Let $\omega(r):(0, \infty) \rightarrow(0, \infty)$ be a measurable function satisfying the doubling condition, that is, there exists a constant $c_{d} \geq 1$ such that

$$
c_{d}^{-1} \omega(r) \leq \omega(t) \leq c_{d} \omega(r) \quad \text { whenever } \quad 0<r<t \leq 2 r .
$$

We consider the variable exponent weighted Lebesgue space

$$
L^{p(\cdot), \omega}\left(\mathbf{R}^{N}\right)=\left\{f \in L_{l o c}^{1}\left(\mathbf{R}^{N}\right):\|f\|_{L^{p(\cdot), \omega}\left(\mathbf{R}^{N}\right)}<\infty\right\},
$$

where

$$
\|f\|_{L^{p(\cdot), \omega}\left(\mathbf{R}^{N}\right)}=\inf \left\{\lambda>0: \int_{\mathbf{R}^{N}} \omega(|x|)\left(\frac{|f(x)|}{\lambda}\right)^{p(x)} d x \leq 1\right\} .
$$

Let $B(x, r)=\left\{y \in \mathbf{R}^{N}:|y-x|<r\right\}$ and $A(r)=B(0,2 r) \backslash B(0, r)$ for $x \in \mathbf{R}^{N}$ and $r>0$. For $q>0$, we consider the variable exponent non-homogeneous central Herz-Morrey space

$$
\mathcal{H}^{p(\cdot), q, \omega}\left(\mathbf{R}^{N}\right)=\left\{f \in L_{\mathrm{loc}}^{1}\left(\mathbf{R}^{N}\right):\|f\|_{\mathcal{H}^{p(\cdot), q, \omega}\left(\mathbf{R}^{N}\right)}<\infty\right\},
$$

where

$$
\|f\|_{\mathcal{H}^{p(\cdot), q, \omega}\left(\mathbf{R}^{N}\right)}=\|f\|_{L^{p(\cdot)}(B(0,2))}+\left(\int_{1}^{\infty}\left(\omega(r)\|f\|_{L^{p(\cdot)}(A(r))}\right)^{q} \frac{d r}{r}\right)^{1 / q}
$$


Lemma 2.1. Let $r_{0}>0$. Let $F(x)$ be a non-negative measurable function on $\mathbf{R}^{N} \backslash B\left(0, r_{0}\right)$ and $v(r)$ be a positive measurable function on $\left[r_{0}, \infty\right)$ satisfying the doubling condition. Then

$$
\begin{aligned}
C^{-1} \int_{\mathbf{R}^{N} \backslash B\left(0,2 r_{0}\right)} v(|x|) F(x) d x & \leq \int_{r_{0}}^{\infty} v(r)\left(\int_{A(r)} F(x) d x\right) \frac{d r}{r} \\
& \leq C \int_{\mathbf{R}^{N} \backslash B\left(0, r_{0}\right)} v(|x|) F(x) d x
\end{aligned}
$$

with a constant $C>0$.

Proof. By the doubling condition on $v$ and Fubini's theorem, we have

$$
\begin{aligned}
& \int_{r_{0}}^{\infty} v(r)\left(\int_{A(r)} F(x) d x\right) \frac{d r}{r} \leq c_{d} \int_{r_{0}}^{\infty}\left(\int_{A(r)} v(|x|) F(x) d x\right) \frac{d r}{r} \\
& \leq c_{d} \int_{\mathbf{R}^{N} \backslash B\left(0, r_{0}\right)} v(|x|) F(x)\left(\int_{|x| / 2}^{|x|} \frac{d r}{r}\right) d x \\
& =(\log 2) c_{d} \int_{\mathbf{R}^{N} \backslash B\left(0, r_{0}\right)} v(|x|) F(x) d x
\end{aligned}
$$

and

$$
\begin{aligned}
& \int_{r_{0}}^{\infty} v(r)\left(\int_{A(r)} F(x) d x\right) \frac{d r}{r} \geq c_{d}^{-1} \int_{r_{0}}^{\infty}\left(\int_{A(r)} v(|x|) F(x) d x\right) \frac{d r}{r} \\
& \geq c_{d}^{-1} \int_{\mathbf{R}^{N} \backslash B\left(0,2 r_{0}\right)} v(|x|) F(x)\left(\int_{|x| / 2}^{|x|} \frac{d r}{r}\right) d x \\
& =(\log 2) c_{d}^{-1} \int_{\mathbf{R}^{N} \backslash B\left(0,2 r_{0}\right)} v(|x|) F(x) d x,
\end{aligned}
$$

as required.

Lemma 2.2. Let $\gamma>0$ and $r_{0} \geq 1$.

(1) If there is $\beta \geq 0$ such that

$$
\int_{A(r)}|f(x)|^{p(x)} d x \leq M r^{\beta} \quad \text { for } r \geq r_{0}
$$

then

$$
\|f\|_{L^{p(\cdot)}(A(r))} \leq r^{-\gamma}+C\left(\int_{A(r)}|f(x)|^{p(x)} d x\right)^{1 / p(\infty)} \quad \text { for } r \geq r_{0}
$$

with a constant $C>0$ depending only on $M, \beta, \gamma$ and $c_{\infty}$.

(2) If there is $\beta^{\prime} \geq 0$ such that

$$
\|f\|_{L^{p(\cdot)}(A(r))} \leq M^{\prime} r^{\beta^{\prime}} \quad \text { for } r \geq r_{0}
$$

then

$$
\int_{A(r)}|f(x)|^{p(x)} d x \leq r^{-\gamma}+C\left(\|f\|_{L^{p(\cdot)}(A(r))}\right)^{p(\infty)} \quad \text { for } r \geq r_{0}
$$

with a constant $C>0$ depending only on $M^{\prime}, \beta^{\prime}, \gamma$ and $c_{\infty}$. 
Proof. Let $\lambda(r)=\|f\|_{L^{p(\cdot)}(A(r))}$. Then

$$
\int_{A(r)}\left(\frac{|f(x)|}{\lambda(r)}\right)^{p(x)} d x=1 .
$$

Assume (2.1). Let $r \geq r_{0}$. If $r^{-\gamma} \leq \lambda(r) \leq 1$, then

$$
C^{-1} \lambda(r)^{-p(\infty)} \leq \lambda(r)^{-p(x)} \leq C \lambda(r)^{-p(\infty)}
$$

for $x \in A(r)$ with a constant $C>0$ depending only on $\gamma$ and $c_{\infty}$. If $\lambda(r) \geq 1$, then $\lambda(r)^{-p(x)} \leq \lambda(r)^{-1}$, so that $\lambda(r) \leq M r^{\beta}$ by (2.3) and (2.1). Hence, for $x \in A(r)$, $\lambda(r)^{-p(x)} \leq C \lambda(r)^{-p(\infty)}$ with a constant $C>0$ depending only on $M, \beta$ and $c_{\infty}$. Hence, by (2.3) again,

$$
\lambda(r) \leq C\left(\int_{A(r)}|f(x)|^{p(x)} d x\right)^{1 / p(\infty)}
$$

in case $\lambda(r) \geq r^{-\gamma}$, which implies the assertion of (1).

The proof of (2) is similar.

Lemma 2.3. If

$$
\int_{1}^{\infty} \omega(r)\left(\|f\|_{L^{p(\cdot)}(A(r))}\right)^{p(\infty)} \frac{d r}{r} \leq 1
$$

then

$$
\int_{\mathbf{R}^{N} \backslash B(0, \sqrt{2})} \omega(|x|)|f(x)|^{p(x)} d x \leq C
$$

with a constant $C<\infty$.

Proof. Suppose (2.4) holds, and set $\lambda(r)=\|f\|_{L^{p(\cdot)}(A(r))}$. First we show

$$
\lambda(r) \leq C \omega(r)^{-1 / p(\infty)} \quad \text { for } r \geq \sqrt{2} .
$$

Set $\mu(r)=\|f\|_{L^{p(\cdot)}(B(0, \sqrt{2} r) \backslash B(0, r))}, r \geq 1$. Since $B(0, \sqrt{2} r) \backslash B(0, r) \subset A(t)$ for $r / \sqrt{2}<$ $t \leq r, \mu(r) \leq \lambda(t)$ for $1 \leq r / \sqrt{2}<t \leq r$, so that

$$
\omega(r) \mu(r)^{p(\infty)} \leq \frac{2 c_{d}}{\log 2} \int_{r / \sqrt{2}}^{r} \omega(t) \lambda(t)^{p(\infty)} \frac{d t}{t} \leq C<\infty
$$

by $(2.4)$. Hence $\mu(r) \leq C \omega(r)^{-1 / p(\infty)}$ for $r \geq \sqrt{2}$. Since $\lambda(r) \leq \mu(\sqrt{2} r)+\mu(r)$, we have (2.5).

Since $\omega(r)$ is assumed to be doubling, there is $\delta>0$ such that

$$
C^{-1} r^{-\delta} \leq \omega(r) \leq C r^{\delta} \quad \text { for } r>1
$$

Let $\gamma>\delta$. Then

$$
\int_{\sqrt{2}}^{\infty} \omega(r) r^{-\gamma} \frac{d r}{r}<\infty
$$

By (2) of the previous lemma (note that (2.5) implies (2.2) for some $\beta^{\prime} \geq 0$ ), we have

$$
\int_{A(r)}|f(x)|^{p(x)} d x \leq r^{-\gamma}+C \lambda(r)^{p(\infty)}
$$


for $r \geq \sqrt{2}$, so that $\int_{A(\sqrt{2})}|f(x)|^{p(x)} d x \leq C$ and

$$
\begin{aligned}
& \int_{\sqrt{2}}^{\infty} \omega(r)\left(\int_{A(r)}|f(x)|^{p(x)} d x\right) \frac{d r}{r} \\
& \leq \int_{\sqrt{2}}^{\infty} \omega(r) r^{-\gamma} \frac{d r}{r}+C \int_{\sqrt{2}}^{\infty} \omega(r)\left(\|f\|_{L^{p(\cdot)}(A(r))}\right)^{p(\infty)} \frac{d r}{r} \\
& \leq C<\infty
\end{aligned}
$$

by (2.7) and (2.4). Hence, by Lemma 2.1, the latter inequalities yield

$$
\int_{\mathbf{R}^{N} \backslash B(0,2 \sqrt{2})} \omega(|x|)|f(x)|^{p(x)} d x \leq C<\infty
$$

and we obtain the assertion of the lemma.

Proposition 2.4. Assume that $0<\inf _{0<r \leq 1} \omega(r) \leq \sup _{0<r \leq 1} \omega(r)<\infty$. Then

$$
C^{-1}\|f\|_{L^{p(\cdot), \omega}\left(\mathbf{R}^{N}\right)} \leq\|f\|_{\mathcal{H}^{p(\cdot), p(\infty), \omega^{1 / p(\infty)}\left(\mathbf{R}^{N}\right)}} \leq C\|f\|_{L^{p(\cdot), \omega}\left(\mathbf{R}^{N}\right)} .
$$

Proof. First, let $\|f\|_{L^{p(\cdot), \omega}\left(\mathbf{R}^{N}\right)} \leq 1$, namely

$$
\int_{\mathbf{R}^{N}} \omega(|x|)|f(x)|^{p(x)} d x \leq 1 .
$$

Then

$$
\int_{B(0,2)}|f(x)|^{p(x)} d x \leq C
$$

and

$$
\int_{A(r)}|f(x)|^{p(x)} d x \leq c_{d} \omega(r)^{-1} \int_{\mathbf{R}^{N}} \omega(|x|)|f(x)|^{p(x)} d x \leq C \omega(r)^{-1}
$$

for $r \geq 1$. Let $\gamma>\delta / p(\infty)$ for $\delta$ in (2.6), so that

$$
\int_{1}^{\infty} \omega(r) r^{-\gamma p(\infty)} \frac{d r}{r}<\infty .
$$

By Lemma $2.2(1)$ and (2.8)

$$
\begin{aligned}
\|f\|_{L^{p(\cdot)}(A(r))} & \leq r^{-\gamma}+C\left(\int_{A(r)}|f(x)|^{p(x)} d x\right)^{1 / p(\infty)} \\
& \leq r^{-\gamma}+C \omega(r)^{-1 / p(\infty)}\left(\int_{A(r)} \omega(|x|)|f(x)|^{p(x)} d x\right)^{1 / p(\infty)}
\end{aligned}
$$

for $r \geq 1$. Therefore, using Lemma 2.1 and (2.9), we have

$$
\begin{aligned}
& \int_{1}^{\infty}\left(\omega(r)^{1 / p(\infty)}\|f\|_{L^{p(\cdot)}(A(r))}\right)^{p(\infty)} \frac{d r}{r} \\
& \leq C\left\{\int_{1}^{\infty} \omega(r) r^{-\gamma p(\infty)} \frac{d r}{r}+\int_{1}^{\infty}\left(\int_{A(r)} \omega(|x|)|f(x)|^{p(x)} d x\right) \frac{d r}{r}\right\} \\
& \leq C\left\{1+\int_{\mathbf{R}^{N} \backslash B(0,1)} \omega(|x|)|f(x)|^{p(x)} d x\right\} \leq C,
\end{aligned}
$$

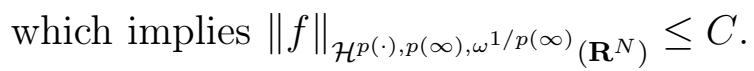


Conversely, suppose $\|f\|_{\mathcal{H}^{p(\cdot), p(\infty), \omega^{1 / p(\infty)}}\left(\mathbf{R}^{N}\right)} \leq 1$, namely

$$
\|f\|_{L^{p(\cdot)}(B(0,2))}+\left(\int_{1}^{\infty} \omega(r)\left(\|f\|_{L^{p(\cdot)}(A(r))}\right)^{p(\infty)} \frac{d r}{r}\right)^{1 / p(\infty)} \frac{d r}{r} \leq 1 .
$$

By Lemma 2.3,

$$
\int_{\mathbf{R}^{N} \backslash B(0, \sqrt{2})} \omega(|x|)|f(x)|^{p(x)} d x \leq C .
$$

Also, $\|f\|_{L^{p(\cdot)(B(0,2))}} \leq 1$ implies

$$
\int_{B(0, \sqrt{2})} \omega(|x|)|f(x)|^{p(x)} d x \leq C .
$$

Thus, $\|f\|_{L^{p(\cdot), \omega}\left(\mathbf{R}^{N}\right)} \leq C$.

For later use we prepare the following result.

Lemma 2.5. There is a constant $C>0$ such that

$$
\frac{1}{|A(r)|} \int_{A(r)}|f(y)| d y \leq C r^{-N / p(\infty)}\|f\|_{L^{p(\cdot)}(A(r))}
$$

for $r>1$.

Proof. Let $r>1$ and $f$ be a nonnegative measurable function on $\mathbf{R}^{N}$ satisfying $\|f\|_{L^{p(\cdot)}(A(r))} \leq 1$. Then

$$
\frac{1}{|A(r)|} \int_{A(r)} f(y) d y \leq|A(r)|^{-1 / p(\infty)}+\frac{1}{|A(r)|} \int_{A(r)} f(y)\left(\frac{f(y)}{|A(r)|^{-1 / p(\infty)}}\right)^{p(y)-1} d y .
$$

Since $|A(r)|^{p(y)} \leq C|A(r)|^{p(\infty)}$ for $y \in A(r)$, we obtain

$$
\begin{aligned}
\frac{1}{|A(r)|} \int_{A(r)} f(y) d y & \leq|A(r)|^{-1 / p(\infty)}+C|A(r)|^{(p(\infty)-1) / p(\infty)} \frac{1}{|A(r)|} \int_{A(r)} f(y)^{p(y)} d y \\
& \leq C|A(r)|^{-1 / p(\infty)},
\end{aligned}
$$

which proves the result.

\section{Norm inequalities for (generalized) Riesz potentials}

For $0<\alpha<N$ and an integer $k \geq 1$, let $I_{\alpha}(x)=|x|^{\alpha-N}$ and

$$
I_{\alpha, k}(x, y)= \begin{cases}I_{\alpha}(x-y) & \text { for }|y|<1 \\ I_{\alpha}(x-y)-\sum_{|\mu| \leq k-1} \frac{x^{\mu}}{\mu !}\left(D^{\mu} I_{\alpha}\right)(-y) & \text { for }|y| \geq 1 .\end{cases}
$$

For $f \in L_{\text {loc }}^{1}\left(\mathbf{R}^{N}\right)$, set

and

$$
I_{\alpha} f(x)=\int_{\mathbf{R}^{N}} I_{\alpha}(x-y) f(y) d y
$$

$$
I_{\alpha, k} f(x)=\int_{\mathbf{R}^{N}} I_{\alpha, k}(x, y) f(y) d y
$$

whenever the integrals are well-defined.

The following estimates are fundamental (see [9], [10] and [15]).

Lemma 3.1. (1) If $2|x|<|y|$ and $|y| \geq 1$, then $\left|I_{\alpha, k}(x, y)\right| \leq C|x|^{k}|y|^{\alpha-N-k}$.

(2) If $|x| / 2 \leq|y| \leq 2|x|$, then $\left|I_{\alpha, k}(x, y)\right| \leq C|x-y|^{\alpha-N}$. 
(3) If $1 \leq|y| \leq|x| / 2$, then $\left|I_{\alpha, k}(x, y)\right| \leq C|x|^{k-1}|y|^{\alpha-N-(k-1)}$.

We consider the following condition $(\mathrm{P})$ for $p(\cdot)$ :

(P) The Hardy-Littlewood maximal operator $M$ is bounded on $L^{p(\cdot)}\left(\mathbf{R}^{N}\right)$.

As is well known, $p(\cdot)$ satisfies $(\mathrm{P})$ if $p^{-}>1$ and $p(\cdot)$ is log-Hölder continuous (locally as well as at $\infty$ ) (see, e.g., [3, Theorem 1.5] and [4, Theorem 4.3.8]). See also $[2,5,6,8]$ for other conditions that guarantee the boundedness of $M$.

We consider the following two types of conditions for $\omega(r)$ :

$(\omega 1 ; \nu) r \mapsto r^{\varepsilon_{1}+\nu} \omega(r)$ is almost decreasing on $[1, \infty)$ for some $\varepsilon_{1}>0$;

$(\omega 2 ; \mu) r \mapsto r^{-\varepsilon_{2}+\mu} \omega(r)$ is almost increasing on $[1, \infty)$ for some $\varepsilon_{2}>0$.

Example 3.2. $\omega(r)=(1+r)^{-a}$ satisfies $(\omega 1 ; \nu)$ if and only if $a>\nu$; it satisfies $(\omega 2 ; \mu)$ if and only if $a<\mu$.

Lemma 3.3. Let $\beta \in \mathbf{R}$. If $\omega(r)$ satisfies $(\omega 1 ; N / p(\infty)-\beta)$, then for $0<\varepsilon<\varepsilon_{1}$

$\int_{B(0, r) \backslash B(0,1)}|y|^{\beta-N}|f(y)| d y \leq C r^{-\varepsilon-N / p(\infty)+\beta} \omega(r)^{-1}\left(\int_{1 / 2}^{r}\left(t^{\varepsilon} \omega(t)\|f\|_{L^{p(\cdot)}(A(t))}\right)^{q} \frac{d t}{t}\right)^{1 / q}$ for all $r \geq 1$ and $f \in L_{l o c}^{1}\left(\mathbf{R}^{N}\right)$.

Proof. We may assume that $f(x)=0$ for $x \in B(0,1)$. Let $j_{0}$ be the smallest integer such that $2^{j_{0}} \geq r$. By Lemma 2.5, we have

$$
\begin{aligned}
\int_{B(0, r) \backslash B(0,1)}|y|^{\beta-N}|f(y)| d y & \leq C \sum_{j=1}^{j_{0}}\left(2^{-j} r\right)^{\beta} \frac{1}{\left|A\left(2^{-j} r\right)\right|} \int_{A\left(2^{-j} r\right)}|f(y)| d y \\
& \leq C \sum_{j=1}^{j_{0}}\left(2^{-j} r\right)^{\beta-N / p(\infty)}\|f\|_{L^{p(\cdot)}\left(A\left(2^{-j} r\right)\right)} .
\end{aligned}
$$

In case $q>1$, by Hölder's inequality and $(\omega 1 ; N / p(\infty)-\beta)$, for $0<\varepsilon<\varepsilon_{1}$, we have

$$
\begin{aligned}
& \sum_{j=1}^{j_{0}}\left(2^{-j} r\right)^{\beta-N / p(\infty)}\|f\|_{L^{p(\cdot)}\left(A\left(2^{-j} r\right)\right)} \\
& \leq\left(\sum_{j=1}^{j_{0}}\left(\left(2^{-j} r\right)^{-\varepsilon+\beta-N / p(\infty)} \omega\left(2^{-j} r\right)^{-1}\right)^{q^{\prime}}\right)^{1 / q^{\prime}} \\
& \cdot\left(\sum_{j=1}^{j_{0}}\left(\left(2^{-j} r\right)^{\varepsilon} \omega\left(2^{-j} r\right)\|f\|_{L^{p(\cdot)}\left(A\left(2^{-j} r\right)\right)}\right)^{q}\right)^{1 / q} \\
& \leq C r^{-\varepsilon_{1}+\beta-N / p(\infty)} \omega(r)^{-1}\left(\sum_{j=1}^{j_{0}}\left(2^{-j} r\right)^{\left(\varepsilon_{1}-\varepsilon\right) q^{\prime}}\right)^{1 / q^{\prime}} \\
& \cdot\left(\sum_{j=1}^{j_{0}}\left(\left(2^{-j} r\right)^{\varepsilon} \omega\left(2^{-j} r\right)\|f\|_{L^{p(\cdot)}\left(A\left(2^{-j} r\right)\right)}\right)^{q}\right)^{1 / q} \\
& \leq C r^{-\varepsilon+\beta-N / p(\infty)} \omega(r)^{-1}\left(\int_{1 / 2}^{r}\left(t^{\varepsilon} \omega(t)\|f\|_{L^{p(\cdot)}(A(t))}\right)^{q} \frac{d t}{t}\right)^{1 / q} .
\end{aligned}
$$

Therefore, we obtain the required result in this case. 
For the case $0<q \leq 1$, by the fact that $(a+b)^{q} \leq a^{q}+b^{q}$ for all $a, b \geq 0$ instead of Hölder's inequality, we also obtain the required result.

Lemma 3.4. Let $\beta \in \mathbf{R}$. If $\omega(r)$ satisfies $(\omega 2 ; N / p(\infty)-\beta)$, then for $0<\varepsilon<\varepsilon_{2}$ $\int_{\mathbf{R}^{N} \backslash B(0, r)}|y|^{\beta-N}|f(y)| d y \leq C r^{\varepsilon-N / p(\infty)+\beta} \omega(r)^{-1}\left(\int_{r / 2}^{\infty}\left(t^{-\varepsilon} \omega(t)\|f\|_{L^{p(\cdot)}(A(t))}\right)^{q} \frac{d t}{t}\right)^{1 / q}$ for all $r \geq 1$ and $f \in L_{\mathrm{loc}}^{1}\left(\mathbf{R}^{N}\right)$.

Proof. We consider only the case $q>1$, since the case $0<q \leq 1$ is easily treated. By Lemma 2.5, Hölder's inequality and $(\omega 2 ; N / p(\infty)-\beta)$, for $0<\varepsilon<\varepsilon_{2}$, we have

$$
\begin{aligned}
& \int_{\mathbf{R}^{N} \backslash B(0, r)}|y|^{\beta-N}|f(y)| d y \leq C \sum_{j=0}^{\infty}\left(2^{j} r\right)^{\beta} \frac{1}{\left|A\left(2^{j} r\right)\right|} \int_{A\left(2^{j} r\right)}|f(y)| d y \\
\leq & C \sum_{j=0}^{\infty}\left(2^{j} r\right)^{\beta-N / p(\infty)}\|f\|_{L^{p(\cdot)}\left(A\left(2^{j} r\right)\right)} \\
\leq & \left(\sum_{j=0}^{\infty}\left(\left(2^{j} r\right)^{\varepsilon+\beta-N / p(\infty)} \omega\left(2^{j} r\right)^{-1}\right)^{q^{\prime}}\right)^{1 / q^{\prime}} \\
& \cdot\left(\sum_{j=0}^{\infty}\left(\left(2^{j} r\right)^{-\varepsilon} \omega\left(2^{j} r\right)\|f\|_{L^{p(\cdot)}\left(A\left(2^{j} r\right)\right)}\right)^{q}\right)^{1 / q} \\
\leq & C r^{\varepsilon_{2}+\beta-N / p(\infty)} \omega(r)^{-1}\left(\sum_{j=0}^{\infty}\left(2^{j} r\right)^{\left(\varepsilon-\varepsilon_{2}\right) q^{\prime}}\right)^{1 / q^{\prime}} \\
& \cdot\left(\sum_{j=0}^{\infty}\left(\left(2^{j} r\right)^{-\varepsilon} \omega\left(2^{j} r\right)\|f\|_{L^{p(\cdot)}\left(A\left(2^{j} r\right)\right)}\right)^{q}\right)^{1 / q} \\
\leq & C r^{\varepsilon+\beta-N / p(\infty)} \omega(r)^{-1}\left(\int_{r / 2}^{\infty}\left(t^{-\varepsilon} \omega(t)\|f\|_{L^{p(\cdot)}(A(t))}\right)^{q} \frac{d t}{t}\right)^{1 / q} .
\end{aligned}
$$

For $\beta \in \mathbf{R}$, let

$$
\omega_{\beta}(r)= \begin{cases}r^{\beta} \omega(r) & \text { for } r \geq 1 \\ \omega(r) & \text { for } 0<r<1 .\end{cases}
$$

Theorem 3.5. Assume that $p(\cdot)$ satisfies $(\mathrm{P})$. If $\omega$ satisfies $(\omega 1 ; N / p(\infty)-N)$ and $(\omega 2 ; N / p(\infty)-\alpha)$, then

$$
\left\|I_{\alpha} f\right\|_{\mathcal{H}^{p(\cdot), q, \omega}-\alpha\left(\mathbf{R}^{N}\right)} \leq C\|f\|_{\mathcal{H}^{p(\cdot), q, \omega}\left(\mathbf{R}^{N}\right)} .
$$

Proof. Let $\|f\|_{\mathcal{H}^{p(\cdot), q, \omega}\left(\mathbf{R}^{N}\right)} \leq 1$ and $f \geq 0$. For $r \geq 2$, set

$$
\begin{aligned}
f & =f \chi_{B(0,1)}+f \chi_{B(0, r / 2) \backslash B(0,1)}+f \chi_{B(0,4 r) \backslash B(0, r / 2)}+f \chi_{\mathbf{R}^{N} \backslash B(0,4 r)} \\
& =f_{0}+f_{1, r}+f_{2, r}+f_{3, r} .
\end{aligned}
$$

Note here that

$$
\int_{B(0,1)} f(y) d y \leq \int_{B(0,1)} d y+\int_{B(0,1)} f(y)^{p(y)} d y \leq C,
$$

so that

$$
I_{\alpha} f_{0}(x) \leq C|x|^{\alpha-N} \leq C r^{\alpha-N}
$$


for $x \in A(r)$. Note from Lemma 2.2 (1) that

$$
\|1\|_{L^{p(\cdot)}(A(r))} \leq C r^{N / p(\infty)} .
$$

Hence

$$
\left\|I_{\alpha} f_{0}\right\|_{L^{p(\cdot)}(A(r))} \leq C r^{\alpha-N}\|1\|_{L^{p(\cdot)}(A(r))} \leq C r^{\alpha-N+N / p(\infty)} .
$$

Using $(\omega 1 ; N / p(\infty)-N)$, we have

$$
\int_{2}^{\infty}\left(r^{-\alpha} \omega(r)\left\|I_{\alpha} f_{0}\right\|_{L^{p(\cdot)}(A(r))}\right)^{q} \frac{d r}{r} \leq C \int_{2}^{\infty}\left(r^{-N+N / p(\infty)} \omega(r)\right)^{q} \frac{d r}{r} \leq C .
$$

Since

$$
I_{\alpha} f_{1, r}(x) \leq C r^{\alpha-N} \int_{B(0, r / 2) \backslash B(0,1)} f(y) d y
$$

for $x \in A(r)$, by Lemma 3.3

$$
I_{\alpha} f_{1, r}(x) \leq C r^{-\varepsilon+\alpha-N / p(\infty)} \omega(r)^{-1}\left(\int_{1 / 2}^{r}\left(t^{\varepsilon} \omega(t)\|f\|_{L^{p(\cdot)}(A(t))}\right)^{q} \frac{d t}{t}\right)^{1 / q}
$$

for $x \in A(r)$ and $0<\varepsilon<\varepsilon_{1}$. Hence, by (3.1),

$$
\left\|I_{\alpha} f_{1, r}\right\|_{L^{p(\cdot)}(A(r))} \leq C r^{-\varepsilon+\alpha} \omega(r)^{-1}\left(\int_{1 / 2}^{r}\left(t^{\varepsilon} \omega(t)\|f\|_{L^{p(\cdot)}(A(t))}\right)^{q} \frac{d t}{t}\right)^{1 / q} .
$$

Therefore,

$$
\begin{aligned}
& \int_{2}^{\infty}\left(r^{-\alpha} \omega(r)\left\|I_{\alpha} f_{1, r}\right\|_{L^{p(\cdot)}(A(r))}\right)^{q} \frac{d r}{r} \\
& \leq C \int_{2}^{\infty} r^{-\varepsilon q}\left(\int_{1 / 2}^{r}\left(t^{\varepsilon} \omega(t)\|f\|_{L^{p(\cdot)}(A(t))}\right)^{q} \frac{d t}{t}\right) \frac{d r}{r} \\
& \leq C \int_{1 / 2}^{\infty}\left(t^{\varepsilon} \omega(t)\|f\|_{L^{p(\cdot)}(A(t))}\right)^{q}\left(\int_{t}^{\infty} r^{-\varepsilon q} \frac{d r}{r}\right) \frac{d t}{t} \\
& \leq C \int_{1 / 2}^{\infty}\left(\omega(t)\|f\|_{L^{p(\cdot)}(A(t))}\right)^{q} \frac{d t}{t} \leq C .
\end{aligned}
$$

Similarly, since

$$
I_{\alpha} f_{3, r}(x) \leq C \int_{\mathbf{R}^{N} \backslash B(0,4 r)}|y|^{\alpha-N} f(y) d y
$$

for $x \in A(r)$, by Lemma 3.4 and (3.1) we have

$$
\left\|I_{\alpha} f_{3, r}\right\|_{L^{p(\cdot)}(A(r))} \leq C r^{\varepsilon^{\prime}+\alpha} \omega(r)^{-1}\left(\int_{r}^{\infty}\left(t^{-\varepsilon^{\prime}} \omega(t)\|f\|_{L^{p(\cdot)}(A(t))}\right)^{q} \frac{d t}{t}\right)^{1 / q}
$$

for $0<\varepsilon^{\prime}<\varepsilon_{2}$. Hence,

$$
\begin{aligned}
& \int_{2}^{\infty}\left(r^{-\alpha} \omega(r)\left\|I_{\alpha} f_{3, r}\right\|_{L^{p(\cdot)}(A(r))}\right)^{q} \frac{d r}{r} \\
& \leq C \int_{2}^{\infty} r^{\varepsilon^{\prime} q}\left(\int_{r}^{\infty}\left(t^{-\varepsilon^{\prime}} \omega(t)\|f\|_{L^{p(\cdot)}(A(t))}\right)^{q} \frac{d t}{t}\right) \frac{d r}{r} \\
& \leq C \int_{2}^{\infty}\left(t^{-\varepsilon^{\prime}} \omega(t)\|f\|_{L^{p(\cdot)}(A(t))}\right)^{q}\left(\int_{2}^{t} r^{\varepsilon^{\prime} q} \frac{d r}{r}\right) \frac{d t}{t} \\
& \leq C \int_{2}^{\infty}\left(\omega(t)\|f\|_{L^{p(\cdot)}(A(t))}\right)^{q} \frac{d t}{t} \leq C .
\end{aligned}
$$


If $x \in A(r)$, then $B(0,4 r) \subset B(x, 6 r)$. Hence

$$
I_{\alpha} f_{2, r}(x) \leq \int_{B(x, 6 r)}|x-y|^{\alpha-N} f_{2, r}(y) d y \leq C r^{\alpha} M f_{2, r}(x)
$$

for $x \in A(r)$. Hence, using $(\mathrm{P})$, we have

$$
\begin{aligned}
\left\|I_{\alpha} f_{2, r}\right\|_{L^{p(\cdot)}(A(r))} & \leq C r^{\alpha}\left\|M f_{2, r}\right\|_{L^{p(\cdot)}(A(r))} \leq C r^{\alpha}\left\|f_{2, r}\right\|_{L^{p(\cdot)}\left(\mathbf{R}^{N}\right)} \\
& \leq C r^{\alpha}\left(\|f\|_{L^{p(\cdot)}(A(r / 2))}+\|f\|_{L^{p(\cdot)}(A(r))}+\|f\|_{L^{p(\cdot)}(A(2 r))}\right),
\end{aligned}
$$

which implies

$$
\int_{2}^{\infty}\left(r^{-\alpha} \omega(r)\left\|I_{\alpha} f_{2, r}\right\|_{L^{p(\cdot)}(A(r))}\right)^{q} \frac{d r}{r} \leq C .
$$

By (3.2), (3.3), (3.4) and (3.5),

$$
\int_{2}^{\infty}\left(r^{-\alpha} \omega(r)\left\|I_{\alpha} f\right\|_{L^{p(\cdot)}(A(r))}\right)^{q} \frac{d r}{r} \leq C .
$$

Finally we obtain

$$
\begin{aligned}
& \left\|I_{\alpha} f\right\|_{L^{p(\cdot)}(B(0,2))}+\left(\int_{1}^{2}\left(r^{-\alpha} \omega(r)\left\|I_{\alpha} f\right\|_{L^{p(\cdot)}(A(r))}\right)^{q} \frac{d r}{r}\right)^{1 / q} \leq C\left\|I_{\alpha} f\right\|_{L^{p(\cdot)}(B(0,4))} \\
& \leq C\left\|I_{\alpha}\left(f \chi_{B(0,8)}\right)\right\|_{L^{p(\cdot)}(B(0,4))}+C\left\|I_{\alpha} f_{3,2}\right\|_{L^{p(\cdot)}(B(0,4))} \leq C .
\end{aligned}
$$

Theorem 3.6. Assume that $p(\cdot)$ satisfies $(\mathrm{P})$. If $\omega$ satisfies $(\omega 1 ; N / p(\infty)-\alpha+$ $k-1)$ and $(\omega 2 ; N / p(\infty)-\alpha+k)$ for an integer $k \geq 1$, then

$$
\left\|I_{\alpha, k} f\right\|_{\mathcal{H}^{p(\cdot), q, \omega_{-\alpha}\left(\mathbf{R}^{N}\right)}} \leq C\|f\|_{\mathcal{H}^{p(\cdot), q, \omega}\left(\mathbf{R}^{N}\right)} .
$$

Proof. Let $\|f\|_{\mathcal{H}^{p(\cdot), q, \omega}\left(\mathbf{R}^{N}\right)} \leq 1$ and $f \geq 0$. For $|x| \geq 2$, set

$$
\begin{aligned}
I_{\alpha, k} f(x)= & I_{\alpha}\left(f \chi_{B(0,1)}\right)(x)+\int_{B(0,|x| / 2) \backslash B(0,1)} I_{\alpha, k}(x, y) f(y) d y \\
& +\int_{B(0,2|x|) \backslash B(0,|x| / 2)} I_{\alpha, k}(x, y) f(y) d y+\int_{\mathbf{R}^{N} \backslash B(0,2|x|)} I_{\alpha, k}(x, y) f(y) d y \\
= & u_{0}(x)+u_{1}(x)+u_{2}(x)+u_{3}(x) .
\end{aligned}
$$

Let $r \geq 2$. In the proof of the previous theorem, we have shown

$$
\left\|u_{0}\right\|_{L^{p(\cdot)}(A(r))} \leq C r^{\alpha-N+N / p(\infty)} .
$$

Since by Lemma 3.1

$$
\begin{aligned}
\left|u_{1}(x)\right| & \leq C|x|^{k-1} \int_{B(0,|x| / 2) \backslash B(0,1)}|y|^{\alpha-N-(k-1)} f(y) d y \\
& \leq C r^{k-1} \int_{B(0, r) \backslash B(0,1)}|y|^{\alpha-N-(k-1)} f(y) d y
\end{aligned}
$$

for $x \in A(r)$, using Lemma 3.3 and (3.1) we have

$$
\left\|u_{1}\right\|_{L^{p(\cdot)}(A(r))} \leq C r^{-\varepsilon+\alpha} \omega(r)^{-1}\left(\int_{1 / 2}^{r}\left(t^{\varepsilon} \omega(t)\|f\|_{L^{p(\cdot)}(A(t))}\right)^{q} \frac{d t}{t}\right)^{1 / q}
$$

for $0<\varepsilon<\varepsilon_{1}$. Similarly, since by Lemma 3.1

$$
\left|u_{3}(x)\right| \leq C|x|^{k} \int_{\mathbf{R}^{N} \backslash B(0,2|x|)}|y|^{\alpha-N-k} f(y) d y \leq C r^{k} \int_{\mathbf{R}^{N} \backslash B(0,2 r)}|y|^{\alpha-N-k} f(y) d y
$$


for $x \in A(r)$, we see by Lemma 3.4 and (3.1),

$$
\left\|u_{3}\right\|_{L^{p(\cdot)}(A(r))} \leq C r^{\varepsilon^{\prime}+\alpha} \omega(r)^{-1}\left(\int_{r}^{\infty}\left(t^{-\varepsilon^{\prime}} \omega(t)\|f\|_{L^{p(\cdot)}(A(t))}\right)^{q} \frac{d t}{t}\right)^{1 / q}
$$

for $0<\varepsilon^{\prime}<\varepsilon_{2}$. Since $\left|u_{2}(x)\right| \leq C I_{\alpha}\left(f \chi_{B(0,4 r) \backslash B(0, r / 2)}\right)(x)$ for $x \in A(r)$ by Lemma 3.1, we have

$$
\left\|u_{2}\right\|_{L^{p(\cdot)}(A(r))} \leq C r^{\alpha}\left(\|f\|_{L^{p(\cdot)}(A(r / 2))}+\|f\|_{L^{p(\cdot)}(A(r))}+\|f\|_{L^{p(\cdot)}(A(2 r))}\right)
$$

as is shown in the proof of the previous theorem. From these estimates, we obtain

$$
\int_{2}^{\infty}\left(r^{-\alpha} \omega(r)\left\|I_{\alpha, k} f\right\|_{L^{p(\cdot)}(A(r))}\right)^{q} \frac{d r}{r} \leq C,
$$

as in the proof of the previous theorem. Finally, noting that $\left|I_{\alpha, k}(x, y)\right| \leq C I_{\alpha}(x-y)$ for $|x| \leq 4$ and $|y| \geq 1$, we obtain

$$
\begin{aligned}
& \left\|I_{\alpha, k} f\right\|_{L^{p(\cdot)}(B(0,2))}+\left(\int_{1}^{2}\left(r^{-\alpha} \omega(r)\left\|I_{\alpha, k} f\right\|_{L^{p(\cdot)}(A(r))}\right)^{q} \frac{d r}{r}\right)^{1 / q} \\
& \leq C\left\|I_{\alpha} f\right\|_{L^{p(\cdot)}(B(0,4))} \leq C,
\end{aligned}
$$

as in the proof of the previous theorem.

Remark 3.7. For $0<\lambda<N / p^{+}$, let $p_{\lambda}(\cdot)$ be defined by

$$
\frac{1}{p_{\lambda}(x)}=\frac{1}{p(x)}-\frac{\lambda}{N}
$$

By modifying the methods expanded in the proof of Theorems 3.5 and 3.6, one can prove: Assume that $p(\cdot)$ satisfies $(\mathrm{P})$. Let $0<\lambda<N / p^{+}$and $\lambda \leq \alpha$.

(1) If $\omega$ satisfies $(\omega 1 ; N / p(\infty)-N)$ and $(\omega 2 ; N / p(\infty)-\alpha)$, then

$$
\left\|I_{\alpha} f\right\|_{\mathcal{H}^{p_{\lambda}(\cdot), q, \omega_{\lambda-\alpha}\left(\mathbf{R}^{N}\right)}} \leq C\|f\|_{\mathcal{H}^{p(\cdot), q, \omega}\left(\mathbf{R}^{N}\right)} .
$$

(2) If $\omega$ satisfies $(\omega 1 ; N / p(\infty)-\alpha+k-1)$ and $(\omega 2 ; N / p(\infty)-\alpha+k)$ for an integer $k \geq 1$, then

$$
\left\|I_{\alpha, k} f\right\|_{\mathcal{H}^{p_{\lambda}(\cdot), q, \omega} \omega_{\lambda-\alpha}\left(\mathbf{R}^{N}\right)} \leq C\|f\|_{\mathcal{H}^{p(\cdot), q, \omega}\left(\mathbf{R}^{N}\right)}
$$

The case $\lambda=\alpha$ obtains the Sobolev type inequality.

Combining Theorems 3.5 and 3.6 with Proposition 2.4, we obtain our main theorem:

Theorem 3.8. Assume that $p(\cdot)$ satisfies $(\mathrm{P})$. Assume that $0<\inf _{0<r \leq 1} \omega(r) \leq$ $\sup _{0<r \leq 1} \omega(r)<\infty$.

(1) If $\omega$ satisfies $(\omega 1 ; N-N p(\infty))$ and $(\omega 2 ; N-\alpha p(\infty))$, then

$$
\left\|I_{\alpha} f\right\|_{L^{p(\cdot), \omega}-\alpha p(\infty)\left(\mathbf{R}^{N}\right)} \leq C\|f\|_{L^{p(\cdot), \omega}\left(\mathbf{R}^{N}\right)} .
$$

(2) If $\omega$ satisfies $(\omega 1 ; N-\alpha p(\infty)+(k-1) p(\infty))$ and $(\omega 2 ; N-\alpha p(\infty)+k p(\infty))$ for an integer $k \geq 1$, then

$$
\left\|I_{\alpha, k} f\right\|_{L^{p(\cdot), \omega}-\alpha p(\infty)\left(\mathbf{R}^{N}\right)} \leq C\|f\|_{L^{p(\cdot), \omega}\left(\mathbf{R}^{N}\right)} .
$$

In case $\omega(r)=(1+r)^{-a}$, we denote $\mathcal{H}^{p(\cdot), q, \omega}\left(\mathbf{R}^{N}\right)$ by $\mathcal{H}^{p(\cdot), q, a}\left(\mathbf{R}^{N}\right)$ and $L^{p(\cdot), \omega}\left(\mathbf{R}^{N}\right)$ by $L^{p(\cdot), a}\left(\mathbf{R}^{N}\right)$. In view of Example 3.2, we have the following corollaries for this special weight:

Corollary 3.9. Assume that $p(\cdot)$ satisfies $(\mathrm{P})$. 
(1) If $N / p(\infty)-N<a<N / p(\infty)-\alpha$, then

$$
\left\|I_{\alpha} f\right\|_{\mathcal{H}^{p(\cdot), q, a+\alpha}\left(\mathbf{R}^{N}\right)} \leq C\|f\|_{\mathcal{H}^{p(\cdot), q, a}\left(\mathbf{R}^{N}\right)} .
$$

(2) If $N / p(\infty)-\alpha+k-1<a<N / p(\infty)-\alpha+k$, then

$$
\left\|I_{\alpha, k} f\right\|_{\mathcal{H}^{p(\cdot), q, a+\alpha}\left(\mathbf{R}^{N}\right)} \leq C\|f\|_{\mathcal{H}^{p(\cdot), q, a}\left(\mathbf{R}^{N}\right)} .
$$

Corollary 3.10. (cf. [7, Theorem B]) Assume that $p(\cdot)$ satisfies $(\mathrm{P})$.

(1) If $N-N p(\infty)<a<N-\alpha p(\infty)$, then

$$
\left\|I_{\alpha} f\right\|_{L^{p(\cdot), a+\alpha p(\infty)}\left(\mathbf{R}^{N}\right)} \leq C\|f\|_{L^{p(\cdot), a}\left(\mathbf{R}^{N}\right)} .
$$

(2) If $N-\alpha p(\infty)+(k-1) p(\infty)<a<N-\alpha p(\infty)+k p(\infty)$ for an integer $k \geq 1$, then

$$
\left\|I_{\alpha, k} f\right\|_{L^{p(\cdot), a+\alpha p(\infty)}\left(\mathbf{R}^{N}\right)} \leq C\|f\|_{L^{p(\cdot), a}\left(\mathbf{R}^{N}\right)} .
$$

\section{The limiting case}

In Corollaries 3.9 and 3.10, there appear conditions that the value of $a$ is in some open intervals. We can show the following in the case $a$ is equal to the lower limiting value.

Proposition 4.1. Assume that $p(\cdot)$ satisfies $(\mathrm{P})$. Let $\delta \geq 1$ and $\delta>1 / q$.

(1) If $a=N / p(\infty)-N$, then

$$
\left\|(\log (2+|\cdot|))^{-\delta} I_{\alpha} f\right\|_{\mathcal{H}^{p(\cdot), q, a+\alpha}\left(\mathbf{R}^{N}\right)} \leq\|f\|_{\mathcal{H}^{p(\cdot), q, a}\left(\mathbf{R}^{N}\right)}
$$

(2) if $a=N / p(\infty)-\alpha+k-1$ for an integer $k \geq 1$, then

$$
\left\|(\log (2+|\cdot|))^{-\delta} I_{\alpha, k} f\right\|_{\mathcal{H}^{p(\cdot), q, a+\alpha}\left(\mathbf{R}^{N}\right)} \leq\|f\|_{\mathcal{H}^{p(\cdot), q, a}\left(\mathbf{R}^{N}\right)} .
$$

Proof of (1). Let $\|f\|_{\mathcal{H}^{p(\cdot), q, a}\left(\mathbf{R}^{N}\right)} \leq 1$ and $f \geq 0$. For $r \geq 2$, set $f=f_{0}+f_{1, r}+$ $f_{2, r}+f_{3, r}$ as in the proof of Theorem 3.5. We know

$$
\left\|I_{\alpha} f_{0}\right\|_{L^{p(\cdot)}(A(r))} \leq C r^{\alpha-N+N / p(\infty)}=C r^{\alpha+a} .
$$

Hence

$$
\begin{aligned}
& \int_{2}^{\infty}\left(r^{-(a+\alpha)}\left\|(\log (2+|\cdot|))^{-\delta} I_{\alpha} f_{0}\right\|_{L^{p(\cdot)}(A(r))}\right)^{q} \frac{d r}{r} \\
& \leq C \int_{2}^{\infty}(\log (2+r))^{-\delta q} \frac{d r}{r}=C<\infty
\end{aligned}
$$

since $\delta>1 / q$. Let $j_{0}$ be the smallest integer such that $2^{j_{0}} \geq r$. For $x \in A(r)$

$$
\begin{aligned}
I_{\alpha} f_{1, r}(x) & \leq C r^{\alpha-N} \int_{B(0, r / 2) \backslash B(0,1)} f(y) d y \\
& \leq C r^{\alpha-N} \sum_{j=2}^{j_{0}}\left(2^{-j} r\right)^{N} \frac{1}{\left|A\left(2^{-j} r\right)\right|} \int_{A\left(2^{-j} r\right)} f(y) d y \\
& \leq C r^{\alpha-N} \sum_{j=2}^{j_{0}}\left(2^{-j} r\right)^{-a}\|f\|_{L^{p(\cdot)}\left(A\left(2^{-j} r\right)\right)}
\end{aligned}
$$

by Lemma 2.5 . 
In case $q>1$, by Hölder's inequality, for $0<\varepsilon<1 / q^{\prime}$, we have

$$
\begin{aligned}
& \sum_{j=2}^{j_{0}}\left(2^{-j} r\right)^{-a}\|f\|_{L^{p(\cdot)}\left(A\left(2^{-j} r\right)\right)} \\
& \leq\left(\sum_{j=2}^{j_{0}}\left(\log \left(2+2^{-j} r\right)\right)^{-\varepsilon q^{\prime}}\right)^{1 / q^{\prime}}\left(\sum_{j=2}^{j_{0}}\left(\left(\log \left(2+2^{-j} r\right)\right)^{\varepsilon}\left(2^{-j} r\right)^{-a}\|f\|_{L^{p(\cdot)}\left(A\left(2^{-j} r\right)\right)}\right)^{q}\right)^{1 / q} \\
& \leq C(\log (2+r))^{-\varepsilon+1 / q^{\prime}}\left(\int_{1 / 2}^{r}\left((\log (2+t))^{\varepsilon} t^{-a}\|f\|_{L^{p(\cdot)}(A(t))}\right)^{q} \frac{d t}{t}\right)^{1 / q} .
\end{aligned}
$$

Therefore, in this case

$$
\begin{aligned}
& (\log (2+r))^{-\delta}\left\|I_{\alpha} f_{1, r}\right\|_{L^{p(\cdot)}(A(r))} \\
& \leq C r^{\alpha-N}(\log (2+r))^{-\varepsilon+1 / q^{\prime}-\delta}\left(\int_{1 / 2}^{r}\left((\log (2+t))^{\varepsilon} t^{-a}\|f\|_{L^{p(\cdot)}(A(t))}\right)^{q} \frac{d t}{t}\right)^{1 / q}\|1\|_{L^{p(\cdot)}(A(r))} \\
& \leq C r^{a+\alpha}(\log (2+r))^{-\varepsilon-1 / q}\left(\int_{1 / 2}^{r}\left((\log (2+t))^{\varepsilon} t^{-a}\|f\|_{L^{p(\cdot)}(A(t))}\right)^{q} \frac{d t}{t}\right)^{1 / q}
\end{aligned}
$$

since $\delta \geq 1$. Thus,

$$
\begin{aligned}
& \int_{2}^{\infty}\left(r^{-(a+\alpha)}\left\|(\log (2+|\cdot|))^{-\delta} I_{\alpha} f_{1, r}\right\|_{L^{p(\cdot)}(A(r))}\right)^{q} \frac{d r}{r} \\
& \leq C \int_{2}^{\infty}(\log (2+r))^{-\varepsilon q-1}\left\{\int_{1 / 2}^{r}(\log (2+t))^{\varepsilon q}\left(t^{-a}\|f\|_{L^{p(\cdot)}(A(t))}\right)^{q} \frac{d t}{t}\right\} \frac{d r}{r} \\
& \leq C \int_{1 / 2}^{\infty}\left(t^{-a}\|f\|_{L^{p(\cdot)}(A(t))}\right)^{q} \frac{d t}{t} \leq C .
\end{aligned}
$$

In case $0<q \leq 1$, (4.1) implies

$$
\begin{aligned}
I_{\alpha} f_{1, r}(x) & \leq C r^{\alpha-N}\left(\sum_{j=2}^{j_{0}}\left(\left(2^{-j} r\right)^{-a}\|f\|_{L^{p(\cdot)}\left(A\left(2^{-j} r\right)\right)}\right)^{q}\right)^{1 / q} \\
& \leq C r^{\alpha-N}\left(\int_{1 / 2}^{r}\left(t^{-a}\|f\|_{L^{p(\cdot)}(A(t))}\right)^{q} \frac{d t}{t}\right)^{1 / q}
\end{aligned}
$$

for $x \in A(r)$. It then follows as above that

$$
\left\|I_{\alpha} f_{1, r}\right\|_{L^{p(\cdot)}(A(r))} \leq C r^{\alpha+a}\left(\int_{1 / 2}^{r}\left(t^{-a}\|f\|_{L^{p(\cdot)}(A(t))}\right)^{q} \frac{d t}{t}\right)^{1 / q} .
$$

Hence,

$$
\begin{aligned}
& \int_{2}^{\infty}\left(r^{-(a+\alpha)}\left\|(\log (2+|\cdot|))^{-\delta} I_{\alpha} f_{1, r}\right\|_{L^{p(\cdot)}(A(r))}\right)^{q} \frac{d r}{r} \\
& \leq C \int_{2}^{\infty}(\log (2+r))^{-\delta q}\left\{\int_{1 / 2}^{r}\left(t^{-a}\|f\|_{L^{p(\cdot)}(A(t))}\right)^{q} \frac{d t}{t}\right\} \frac{d r}{r} \\
& \leq C\left(\int_{1 / 2}^{\infty}\left(t^{-a}\|f\|_{L^{p(\cdot)}(A(t))}\right)^{q} \frac{d t}{t}\right) \int_{1 / 2}^{\infty}(\log (2+r))^{-\delta q} \frac{d r}{r} \\
& \leq C \int_{1 / 2}^{\infty}\left(t^{-a}\|f\|_{L^{p(\cdot)}(A(t))}\right)^{q} \frac{d t}{t} \leq C,
\end{aligned}
$$


since $\delta q>1$.

For $I_{\alpha} f_{2, r}$ and $I_{\alpha} f_{3, r}$, we have the same estimates as in the proof of Theorem 3.5. Thus, we obtain

$$
\int_{2}^{\infty}\left(r^{-(a+\alpha)}\left\|(\log (2+|\cdot|))^{-\delta} I_{\alpha} f\right\|_{L^{p(\cdot)}(A(r))}\right)^{q} \frac{d r}{r} \leq C .
$$

The final part of the proof is the same as that of the proof of Theorem 3.5.

Proof of (2). Let $\|f\|_{\mathcal{H}^{p(\cdot), q, a}\left(\mathbf{R}^{N}\right)} \leq 1$ and $f \geq 0$. For $|x| \geq 2$, set

$$
I_{\alpha, k} f(x)=u_{0}(x)+u_{1}(x)+u_{2}(x)+u_{3}(x)
$$

as in the proof of Theorem 3.6. Let $r \geq 2$. We have shown

$$
\left\|u_{0}\right\|_{L^{p(\cdot)}(A(r))} \leq C r^{\alpha-N+N / p(\infty)}=C r^{a+\alpha-(N-\alpha+k-1)} .
$$

Since $N-\alpha+k-1>0$,

$$
\int_{2}^{\infty}\left(r^{-(a+\alpha)}\left\|u_{0}\right\|_{L^{p(\cdot)}(A(r))}\right)^{q} \frac{d r}{r} \leq C .
$$

Next, for $x \in A(r)$, we have by Lemmas 3.1 and 2.5

$$
\begin{aligned}
\left|u_{1}(x)\right| & \leq C|x|^{k-1} \int_{B(0, r) \backslash B(0,1)}|y|^{\alpha-N-(k-1)} f(y) d y \\
& \leq C r^{k-1} \sum_{j=1}^{j_{0}}\left(2^{-j} r\right)^{\alpha-(k-1)} \frac{1}{\left|A\left(2^{-j} r\right)\right|} \int_{A\left(2^{-j} r\right)} f(y) d y,
\end{aligned}
$$

where $j_{0}$ is the smallest integer such that $2^{j_{0}} \geq r$.

In case $q>1$, as in the proof of (1), we see

$$
\begin{aligned}
\left|u_{1}(x)\right| & \leq C r^{k-1} \sum_{j=1}^{j_{0}}\left(2^{-j} r\right)^{-a}\|f\|_{L^{p(\cdot)}\left(A\left(2^{-j} r\right)\right)} \\
& \leq C r^{k-1}(\log (2+r))^{-\varepsilon+1 / q^{\prime}}\left(\int_{1 / 2}^{r}\left((\log (2+t))^{\varepsilon} t^{-a}\|f\|_{L^{p(\cdot)}(A(t))}\right)^{q} \frac{d t}{t}\right)^{1 / q}
\end{aligned}
$$

for $0<\varepsilon<1 / q^{\prime}$. Since $k-1+N / p(\infty)=a+\alpha$, it follows that

$$
\int_{2}^{\infty}\left(r^{-(a+\alpha)}\left\|(\log (2+|\cdot|))^{-\delta} u_{1}\right\|_{L^{p(\cdot)}(A(r))}\right)^{q} \frac{d r}{r} \leq C
$$

as in the proof of (1). The case $0<q \leq 1$ can be treated in the same way as in the proof of (1).

For the rest of the proof, we can use the same estimates as given in the proof of Theorem 3.6.

By Proposition 2.4, we have the following:

Proposition 4.2. Assume that $p(\cdot)$ satisfies $(\mathrm{P})$. Let $\delta \geq 1$.

(1) If $a=N-N p(\infty)$, then

$$
\left\|(\log (2+|\cdot|))^{-\delta} I_{\alpha} f\right\|_{L^{p(\cdot), a+\alpha p(\infty)}\left(\mathbf{R}^{N}\right)} \leq C\|f\|_{L^{p(\cdot), a}\left(\mathbf{R}^{N}\right)} .
$$

(2) If $a=N-\alpha p(\infty)+(k-1) p(\infty)$ for an integer $k \geq 1$, then

$$
\left\|(\log (2+|\cdot|))^{-\delta} I_{\alpha, k} f(x)\right\|_{L^{p(\cdot), a+\alpha p(\infty)}\left(\mathbf{R}^{N}\right)} \leq C\|f\|_{L^{p(\cdot), a}\left(\mathbf{R}^{N}\right)} .
$$




\section{References}

[1] Capone, C., D. Cruz-Uribe, and A. Fiorenza: The fractional maximal operator and fractional integrals on variable $L^{p}$ spaces. - Rev. Mat. Iberoam. 23:3, 2007, 743-770.

[2] Cruz-Uribe, D., and A. Fiorenza: Variable Lebesgue spaces. Foundations and harmonic analysis. - Appl. Numer. Harmon. Anal., Birkhauser/Springer, Heidelberg, 2013.

[3] Cruz-Uribe, D., A. Fiorenza, and C. J. Neugebauer: The maximal function on variable $L^{p}$ spaces. - Ann. Acad. Sci. Fenn. Math. 28, 2003, 223-238; Ann. Acad. Sci. Fenn. Math. 29, 2004, 247-249.

[4] Diening, L., P. Harjulehto, P. HÄstö and M. RŮžıČKa: Lebesgue and Sobolev spaces with variable exponents. - Lecture Notes in Math. 2017, Springer, Heidelberg, 2011.

[5] Izuki, M., E. Nakai, and Y. Sawano: The Hardy-Littlewood maximal operator on Lebesgue spaces with variable exponent. - RIMS Kôkyûroku Bessatsu B42, 2013, 51-94.

[6] IzUKI, M., E. NAKAI, and Y. SAWANo: Function spaces with variable exponents. An introduction. - Sci. Math. Jpn. 77, 2014, 187-315.

[7] Kurokawa, T.: A weighted norm inequality for potentials of order $(m, k)$. - J. Math. Kyoto Univ. 26-2, 1986, 203-211.

[8] Lerner, A. K.: Some remarks on the Hardy-Littlewood maximal function on variable $L^{p}$ spaces. - Math. Z. 251:3, 2005, 509-521.

[9] Mizuta, Y.: Potential theory in Euclidean spaces. - Gakkōtosho, Tokyo, 1996.

[10] Mizuta, Y.: Integral representations, differentiability properties and limits at infinity for Beppo Levi functions. - Potential Anal. 6, 1997, 237-276.

[11] Mizuta, Y., and T. Shimomura: Weighted Sobolev inequality in Musielak-Orlicz space. - J. Math. Anal. Appl. 388, 2012, 86-97.

[12] Mizuta, Y., and T. Shimomura: Weighted Morrey spaces of variable exponent and Riesz potentials. - Math. Nachr. 288, 2015, 984-1002.

[13] Muckenhoupt, B., and R. L. Wheeden: Weighted norm inequalities for fractional integrals. - Trans. Amer. Math. Soc. 192, 1974, 261-274.

[14] Samko, N., S. Samko, and B. Vakulov: Weighted Sobolev theorem in Lebesgue spaces with variable exponent. - J. Math. Anal. Appl. 335, 2007, 560-583.

[15] Shimomura, T., and Y. Mizuta: Taylor expansion of Riesz potentials. - Hiroshima Math. J. $25,1995,595-621$.

Received 1 December 2016 • Accepted 23 November 2017 